\title{
Geometric Correction Algorithm for UAV Remote Sensing Image Based on Neural Network
}

\author{
Lirong Diao ${ }^{a}$, Riuan Liu ${ }^{\mathrm{b}}$ and Tingting Chen ${ }^{\mathrm{c}}$ \\ School of electronic and communication engineering, Tianjin Normal University, Tianjin 300387, \\ China.

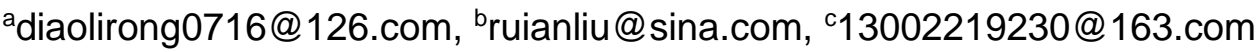

\begin{abstract}
In the process of the UAV (Unmanned Aerial Vehicle) remote sensing image geometric correction, the method of the geometric correction plays a vital role. Since the neural network is a distributed and parallel mathematical model, and it has good learning ability for nonlinear, so the nonlinear and uncertainty of the UVA remote sensing image geometric correction can be solved well. This paper focuses on the application of BP neural network and RBF neural network in UAV remote sensing image geometric correction, and finally compares the effect of the geometric correction based on BP neural network and RBF neural network through the experiments.
\end{abstract}

Keywords: UAV remote sensing image; Neural network; Geometric correction.

\section{Introduction}

As a branch of remote sensing systems, UAV remote sensing system develops fairly rapidly. UAV remote sensing system has many obvious advantages such as high flexibility, targeted, low cost, and so on. Therefore, UAV remote sensing system can provide information for many areas such as urban construction, mapping, environmental monitoring and so on [1]. However, due to the small volume, weak wind resistance and poor stability of UAV, the images will be distorted during shot by UAV, so the geometric correction is needed for distorted UAV remote sensing image.

In the UAV remote image geometric correction method, the core is to establish the geometric correction model. Traditional UAV remote sensing image geometric correction methods such as polynomial correction method and collinear equation correction method are based on the algorithm of least square, and as the principle of experience minimization, so there will be some problems such as weak generalization ability, over learning and other issues. Neural networks are very suitable for fitting non-linear function rely on the strong nonlinear learning ability [2]. Compared to the traditional geometric correction algorithms, the algorithm based on neural network needn't establish a nonlinear function, it only establishes the network structure for geometric correction through the adjustment process.

This paper will study the geometric correction algorithm for UAV remote sensing image based on BP and RBF neural network, and compare the correction effect through experiments, so as to improve the neural network correction capabilities further which are used in the UAV remote sensing image geometric correction.

\section{Geometric Correction Algorithm for UAV Remote Sensing Image Based on BP Neural Network}

\subsection{The Theory of BP Neural Network}

BP neural network is the multi-layer network training as the error back propagation, whose basic principle is that through errors of reverse spreading to adjust the network weights and thresholds constantly so that the square error of the network is minimal. A typical three-layer BP neural network structure is shown in Fig.1. 


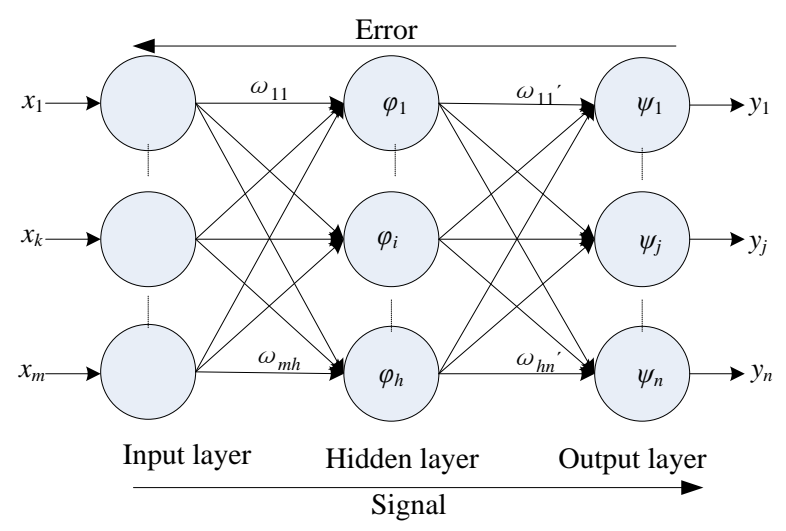

Fig. 1 The structure of BP neural network

In BP neural network, set $\omega_{k i}$ as connection weights between the input layer and the hidden layer, $a_{i}$ as the threshold value of node $i$ in the hidden layer, $\omega_{i j}$ ' as connection weights between the hidden layer and the output layer, and $b_{j}$ as the threshold value of node $j$ in the output layer, $k=1,2, \ldots, m$, $i=1,2, \ldots, h, j=1,2, \ldots, n$ are the input layer, the hidden layer and the number of output layer nodes:

1) In hidden layers, the input net $t_{i}$ and the output $\varphi(i)$ of node $i$ can be expressed as equation (1) and equation (2), where $\varphi(\cdot)$ is the activation function of hidden layers.

$n e t_{i}=\sum_{k=1}^{m} \omega_{k i} x_{k}+a_{i}$

$\varphi(i)=\varphi\left(n e t_{i}\right)=\varphi\left(\sum_{k=1}^{m} \omega_{k i} x_{k}+a_{i}\right)$

2) In output layers, the input net ' ' and the output $O_{j}$ of node $j$ can be expressed as equation (3) and equation $(4)$, where $\psi(\cdot)$ is the activation function of output layers.

$$
\begin{aligned}
& n e t_{j}^{\prime}=\sum_{i=1}^{h} \omega_{i j} \varphi(i)+b_{j}=\sum_{i=1}^{h} \omega_{i j} \varphi\left(\sum_{k=1}^{m} \omega_{k i} x_{k}+a_{i}\right)+b_{j} \\
& O_{j}=\psi\left(n e t_{j}^{\prime}\right)=\psi\left(\sum_{i=1}^{h} \omega_{i j} \varphi\left(\sum_{k=1}^{m} \omega_{k i} x_{k}+a_{i}\right)+b_{j}\right)
\end{aligned}
$$

In BP neural network, the process of error back propagation commonly used the error gradient descent method to adjust the value of each layer, until the error between the value of actual output and the desired output meets the requirements so far [3].

\subsection{The Thought of Geometric Correction Algorithm for UAV Remote Sensing Image Based on BP Neural Network}

In applying the geometric correction algorithm for UAV remote sensing image based on BP neural network, input and desired output should be set by the control point information firstly, the follow is the thought of geometric correction algorithm for UAV remote sensing image based on BP neural network:

Step1: Set $\mathrm{P}=\left(x_{1}, x_{2}, \ldots, x_{m}\right)$ as the control point coordinates of the distorted image, and $\mathrm{T}=\left(x_{1}{ }^{\prime}, x_{2}, \ldots, x_{m}{ }^{\prime}\right)$ as the control point coordinates of the reference image corresponding to the distorted image, then set $\mathrm{P}$ and $\mathrm{T}$ as the input sample and the desired output samples respectively of BP neural network.

Step2: Select the relevant parameters for the base function. Determine the number of units in hidden layers, and randomly select the initial $\omega_{k i}, \omega_{i j}$ ' and $a_{i}, b_{j}$.

Step3: Input P to BP network, calculate the real output $O_{j}$ of the network according to Equation (4),then combine with $\mathrm{T}$, adjust the weights and thresholds of the network using the gradient descent method until the resulting error satisfies the requirement.

Step4: When determining the specific parameters of BP neural network, using the network to do geometric correction for UAV remote sensing images, then do pixel re-sampling, finally obtaining the images after geometric correction. 


\section{Geometric Correction Algorithm for UAV Remote Sensing Image Based on RBF Neural Network}

\subsection{The Theory of RBF Neural Network}

RBF neural network is a front neural network, including input layer, hidden layer and output layer. $\mathrm{RBF}$ neural network can approximate any continuous function with arbitrary precision, it has simple network structure and global optimum and optimum performance, so it can satisfy the UAV remote sensing image geometric correction function nonlinearity and uncertainty well. A RBF neural network is shown in Fig.2.

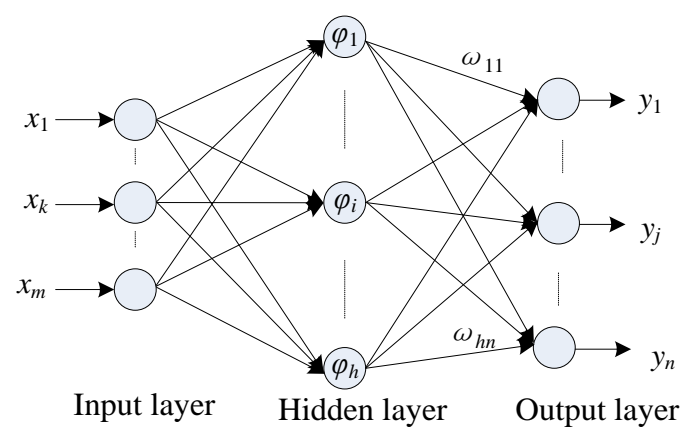

Fig.2 The structure of RBF neural network

The activation function of the hidden layer in RBF neural network is RBF, and usually select Guassian function as the activation function, it expresses as:

$$
\varphi_{i}(x)=\exp \left(-\frac{1}{2 \sigma_{i}^{2}}\left\|x-c_{i}\right\|\right), i=1,2, \ldots, h
$$

Where, $x=\left(x_{1}, x_{2}, \ldots, x_{m}\right)^{\mathrm{T}}$ is the input vector, $\varphi_{i}(x)$ is the output of the node $i$ in the hidden layer, $c_{i}$ is the center value of Guassian function, $\sigma_{i}$ is the variance, and $h$ is the number of the hidden layer units.

The output of the output layer can be expressed as:

$$
y_{j}=\sum_{i=1}^{h} \omega_{i j} \varphi_{i}(x), j=1,2, \ldots, n
$$

Where, $y_{j}$ is the output of the node $j$ in the output layer, $\omega_{i j}$ is connection weights between the hidden layer and the output layer.

In the RBF neural networks, it usually adopts self-organizing method to certain $c_{i}$ and $\sigma_{i}$ of the network, and in this method, k-means algorithm and LMS algorithm are respectively used to determine $c_{i}$ and $\sigma_{i}$.

\subsection{The Thought of Geometric Correction Algorithm for UAV Remote Sensing Image Based on RBF Neural Network}

Using the information of ground control points, and combining with self-organizing method, the thought of geometric correction algorithm for UAV remote sensing image based on RBF neural network is as follows:

Step1: Set $\mathrm{P}=\left(x_{1}, x_{2}, \ldots, x_{m}\right)$ as the control point coordinates of the distorted image, and $\mathrm{T}=\left(x_{1}{ }^{\prime}, x_{2}, \ldots, x_{m}{ }^{\prime}\right)$ as the control point coordinates of the reference image corresponding to the distorted image, then set $\mathrm{P}$ and $\mathrm{T}$ as the input sample and the desired output samples respectively of RBF neural network.

Step2: Select the relevant parameters for the base function. Using k- means algorithm to select the centers $c_{i}$ of the base function in the hidden layer units, then according to equation (7) to calculate the variance $\sigma_{i}$

$$
\sigma_{i}=\frac{C_{\max }}{\sqrt{2 m}}, i=1,2, \ldots, m
$$

Step3: Calculate the actual output $\mathrm{Y}$ according to equation(6), then combine $\mathrm{T}$ to adapt the connection weights $\omega_{i j}$ using LMS algorithm . 
Step4: When determining the specific parameters of RBF neural network, using the network to do geometric correction for UAV remote sensing images, then do pixel re-sampling, finally obtaining the images after geometric correction.

\section{The Comparison and Analysis of the Algorithms}

In order to compare the algorithms for UAV remote sensing image geometric correction, the paper uses the simulation board diagram to test them, and it shows in Fig.3. Where, (a) is the reference image without distortion, its size is 450pixel $\times 450$ pixel, (b) is distorted image, (c) is the corrected image which is used the geometric correction algorithm based on BP neural network, and (d) is the corrected image which is used the geometric correction algorithm based on RBF neural network.

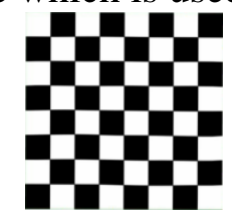

(a)

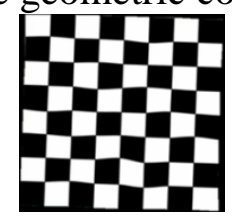

(b)

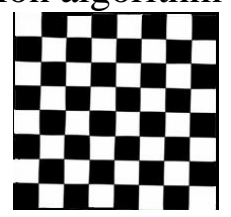

(c)

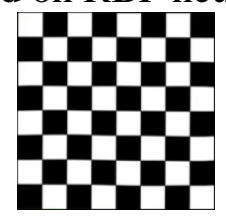

(d)

Fig.3 The simulation image for the experiment

Before doing geometric correction for the distorted image, the control points should be extracted from the image firstly. This paper selects 64 control points, to train the network. Finally, using the trained network to do geometric correction for the distorted image, and correction results are shown in fig.3(c) and fig. 3(d). In order to objectively compare the effect of the two algorithms, 8 points are randomly selected from the corrected images to test the performance of the networks, and the results show in Table1 and Table2.

Table 1 Results of geometric correction algorithms based on BP neural network

\begin{tabular}{ccccc}
\hline $\begin{array}{c}\text { The number } \\
\text { of the points }\end{array}$ & $\begin{array}{c}\text { The points of } \\
\text { reference image }\end{array}$ & $\begin{array}{c}\text { The points of } \\
\text { distorted image }\end{array}$ & $\begin{array}{c}\text { The points of } \\
\text { corrected image }\end{array}$ & RMSE \\
\hline 1 & $(114.00,58.25)$ & $(128.00,65.00)$ & $(112.75,57.06)$ & 1.2204 \\
2 & $(225.00,50.25)$ & $(232.00,57.75)$ & $(225.00,51.00)$ & 0.5303 \\
3 & $(282.00,113.00)$ & $(283.00,122.00)$ & $(283.00,111.38)$ & 1.3462 \\
4 & $(170.00,169.30)$ & $(176.00,175.00)$ & $(170.50,169.82)$ & 0.5101 \\
5 & $(226.00,225.00)$ & $(227.00,226.00)$ & $(224.34,224.50)$ & 1.2259 \\
6 & $(57.00,282.00)$ & $(67.00,275.00)$ & $(57.21,280.75)$ & 0.8963 \\
7 & $(338.00,338.00)$ & $(328.50,335.75)$ & $(336.86,339.08)$ & 1.1104 \\
8 & $(169.00,348.00)$ & $(170.00,340.00)$ & $(170.07,347.23)$ & 0.9321 \\
\hline
\end{tabular}

Table 2 Results of geometric correction algorithms based on RBF neural network

\begin{tabular}{ccccc}
\hline $\begin{array}{c}\text { The number } \\
\text { of the points }\end{array}$ & $\begin{array}{c}\text { The points of } \\
\text { reference image }\end{array}$ & $\begin{array}{c}\text { The points of } \\
\text { distorted image }\end{array}$ & $\begin{array}{c}\text { The points of } \\
\text { corrected image }\end{array}$ & RMSE \\
\hline 1 & $(114.00,58.25)$ & $(128.00,65.00)$ & $(115.00,58.00)$ & 0.7289 \\
2 & $(225.00,50.25)$ & $(232.00,57.75)$ & $(227.00,50.00)$ & 1.4252 \\
3 & $(282.00,113.00)$ & $(283.00,122.00)$ & $(283.00,113.15)$ & 0.7150 \\
4 & $(170.00,169.30)$ & $(176.00,175.00)$ & $(170.50,169.00)$ & 0.4123 \\
5 & $(226.00,225.00)$ & $(227.00,226.00)$ & $(226.00,224.50)$ & 0.3536 \\
6 & $(57.00,282.00)$ & $(67.00,275.00)$ & $(58.00,280.75)$ & 1.1319 \\
7 & $(338.00,338.00)$ & $(328.50,335.75)$ & $(336.50,338.75)$ & 1.1859 \\
8 & $(169.00,348.00)$ & $(170.00,340.00)$ & $(170.00,347.50)$ & 0.7906 \\
\hline
\end{tabular}

From Table1, Table2 and Fig.3, it can obtain that geometric correction algorithms for image based on BP neural network and RBF neural network both have good effects. Analyzing RMSE, it can be seen that the latter's is smaller overall, in other words, the correction accuracy of the image is higher. However, during the experiment, the former corrected faster. 


\section{Summary}

This paper analyzes the current research status of geometric correction for UAV remote sensing image, introduces BP neural network and RBF neural network, and makes a detailed description of geometric correction algorithm for UAV remote sensing image based on BP neural network and RBF neural network. Finally, the paper makes the experiments to compare and analysis their correction effects. It can be seen from the experimental results, as to geometric correction algorithm based on BP neural network and RBF neural network, they both have their own advantages: the latter has a higher correction accuracy, but the former's correct speed is faster. For the optimization algorithm of network parameters, it remains to be further studied.

\section{Acknowledgments}

This research was supported by the Tianjin natural science fund (13JCYBJC15800).

\section{References}

[1] Guo Dong: Research about the registration method of UAV remote sensing image for disaster investigation. University of electronic science and technology of China, 2011.p.1.

[2] Lu Tongtong: Research on neural network of non-linear image distortion correction and identification technology. China Jiliang University, 2013.p.2.

[3] Liu Caihong: The study of algorithm of BP neural network. Chongqing normal university, 2008.p.14. 